\title{
First heterozygous NOP10 mutation in familial pulmonary fibrosis
}

\author{
To the Editor:
}

Germline telomere-related gene mutations are associated with familial pulmonary fibrosis and lead to short telomere syndrome $[1,2]$. Most of the short telomere syndrome-related genes, such as TERT, TERC, DKC1, TINF2, NHP2 and NOP10, were identified in dyskeratosis congenita patients with early onset mucocutaneous manifestations and/or bone marrow failure [3]. The telomerase complex includes TERT, the TERC RNA and DKC1. NOP10, along with DKC1, NHP2 and GAR1, is essential for TERC stability and telomere maintenance [4]. Homozygous NOP10 mutation (c.100C > T, p.Arg34Trp) has been reported only once in a consanguineous family with autosomic recessive dyskeratosis congenita without pulmonary fibrosis [5]. Moreover, the p.Arg34Trp NOP10 mutation caused telomere shortening in homozygous and heterozygous carriers [5]. Here we provide evidence that a heterozygous NOP10 mutation (c.17A>G,p. Tyr6Cys) identified in a large family co-segregates with adult-onset familial pulmonary fibrosis.

The proband (II.1), a 68-year-old non-smoker female, was diagnosed with pulmonary fibrosis at the age of 66 years (figure 1a and b). Two deceased, a brother (II.2) and sister (II.6), and one alive sister (II.7) were also diagnosed with pulmonary fibrosis (figure 1c). Another brother (II.4) and his son (III.5) died from leukaemia while one of the sons of the proband (III.2) had long-term leukopenia. The deceased father of the proband (I.1) was diagnosed with non-alcoholic liver cirrhosis (figure 1a). Mucocutaneous manifestations were not observed upon examination in this family.

We identified by whole exome sequencing in the proband (II.1) a heterozygous pathogenic missense mutation in NOP10 (NM_ 018648) c.17A >G,p.Tyr6Cys according to international recommendations (figure 1a) [6]. No other rare variants predicted to be deleterious were found in whole exome data from the proband in the other genes linked to short telomere syndrome (TERT, TERC, DKC1, TINF2, RTEL1, PARN, NAF1, ACD, NHP2, WRAP53, SHQ1 and ZCCHC8) [7-9]. The variation is absent in 140000 individuals from the gnomAD database. The mutated adenine is conserved at a genomic level $(\mathrm{GERP}=5)$. The in silico tools Polyphen 2 and CADD predicted a deleterious impact of the amino acid change $\mathrm{p}$. Tyr6Cys with scores of 1 and 24, respectively, reflecting high conservation at a protein level. Flow cytometry and FISH (flowFISH) revealed that telomere length of the proband were shorter than the first percentile [6]. It was not possible to organise flowFISH analysis for all individuals of this family. Thus, telomere length for the individuals II.1, II.7, III.1 and III.2 was also measured through telomeric restriction fragment assay [9] at $7.4 \mathrm{~kb}, 6.3 \mathrm{~kb}, 8.5 \mathrm{~kb}$ and $8.6 \mathrm{~kb}$, respectively. Telomere length for the individuals III.1 and III.2 were found to be shorter relative to the mother's telomere length [2]. The fact that non-carrier (III.1) and carrier (III.2) brother were found to have the same range of telomere length could be attributed to the "heritability" of telomere length and evocates epigenetic inheritance [10]. The variation co-segregated with pulmonary fibrosis (II.1 II.7; and the obligate carrier II.6) and leukopenia (III.2). Incomplete penetrance observed for the asymptomatic niece (III.8) carrier of the mutation is frequently observed in short telomere syndrome at the age of 50 years (figure 1a) [2].

Analysis of the human NOP10 protein three-dimensional structure demonstrated that the mutated amino acid Tyr6 is buried with the small, N-terminal globular, ribbon domain of NOP10, which is in close contact with Dyskerin DKC1 (Cbf5 in the archaeal complex) (figure 1d). Hence it is likely that it plays a critical role for the stability of the domain as well as for the integrity of the DKC1/NHP2/GAR1/NOP10 complex [11-14].

@ERSpublications

We provide first evidence that a heterozygous NOP10 mutation (c.17A>G,p.Tyr6Cys) identified in a large family co-segregates with adult-onset familial PF and predisposes to short telomere syndrome (familial PF, liver, haematological diseases) http://bit.ly/2wvXsUd

Cite this article as: Kannengiesser C, Manali ED, Revy P, et al. First heterozygous NOP10 mutation in familial pulmonary fibrosis. Eur Respir J 2020; 55: 1902465 [https://doi.org/10.1183/13993003.02465-2019]. 

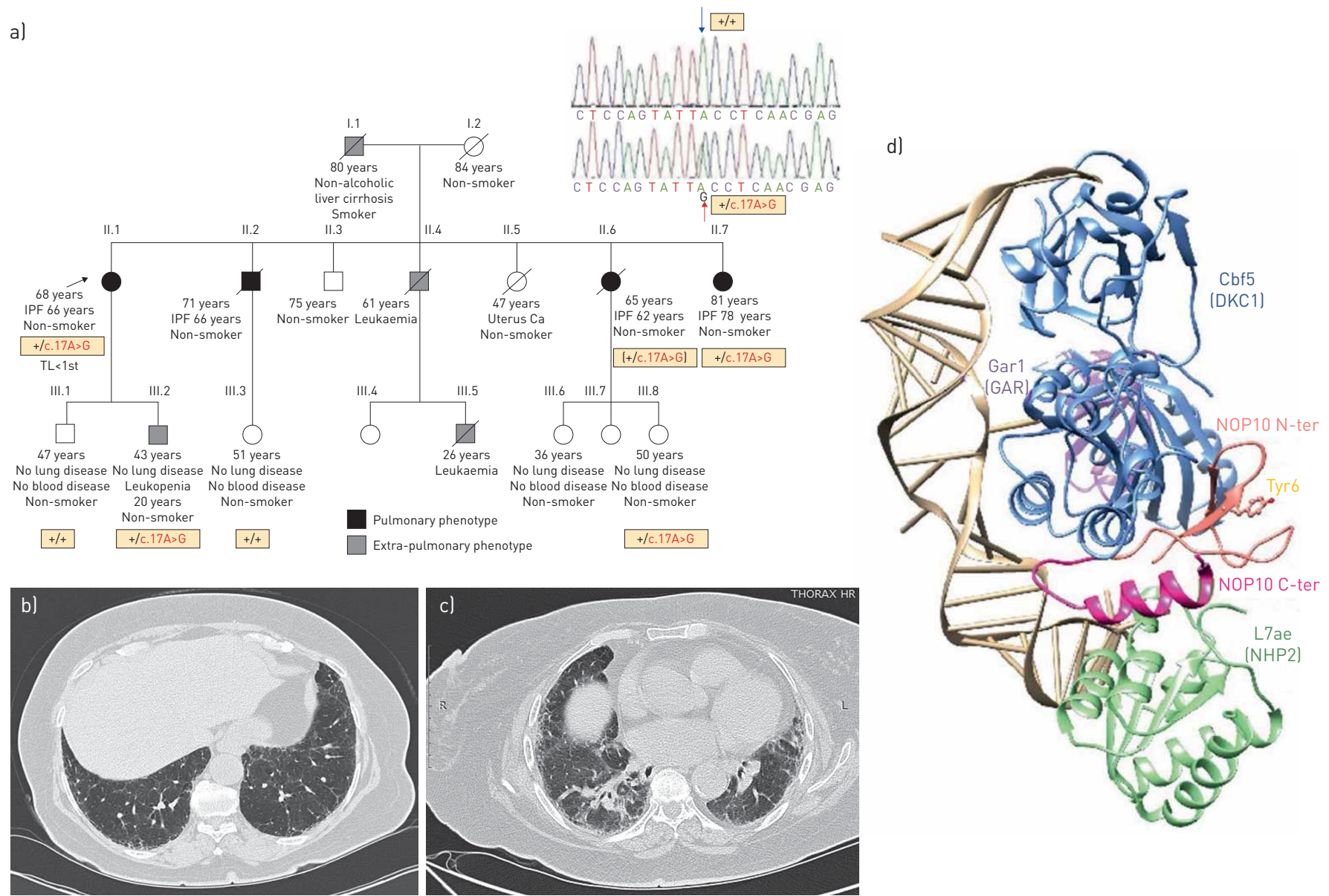

FIGURE 1 a) Three-generation pedigree of the proband. Numbers below individual symbols are age at death or study. NOP10 mutation status in subjects for whom DNA was available for sequencing and pulmonary and extra-pulmonary phenotype is also depicted. (+/c.17A>G) discloses obligate carrier state. In the insert, the presence of NOP10 mutation identified by whole exome sequencing was confirmed by Sanger sequencing. The symbol +/+ discloses wild type and +/c.17A>G heterozygosity for the mutation. TL: telomere length by flowFISH. b) High-resolution computed tomography (HRCT) image at the basal segments of the lower lobes of the proband (II.1) showing peripheral fine reticulation with a microcystic pattern subpleurally. In the inner lung zones intralobular reticulation in association with thin wall traction bronchiectasis are depicted. All features indicate the fibrotic nature of the process. c) HRCT of the chest at the level of the basal segments of lower lobes of the patient II.7 revealing definite reticulation subpleurally with areas of increased density resulting from coalescence of the reticular opacities and areas of microatelectasis. Reticular changes are also seen more centrally distorting the shape of the vessels, while few areas of macrocystic (honeycomb) changes are seen in contact with the mediastinal pleura. The lungs bilaterally are ill expanded (small volumes). d) Ribbon representation of the model of human NOP10, within the frame of a whole H/ACA RNP complex lexperimental three-dimensional structure from Pyroccoccus furiosus, PDB 2EY4). A model of the three-dimensional structure of human NOP10 has been made considering, for its N-terminal region (aa 1-33), the experimental three-dimensional structure of Saccharomyces cerevisiae Nop10 (solved in complex with Cbf5/Dkc1 and Gar1 (pdb 3U28 [11]) and, for the C-terminal region (aa 34-65), the experimental 3D structure of Pyrococcus furiosus Nop10, folded in part into an alpha-helix when contacting both Cbf5 and L7ae/Nhp2 (pdb 2EY4 [12]). The three-dimensional structure of human NOP10 could not be modelled as a whole starting from the archaeal Nop10 three-dimensional structure, as the $\mathrm{N}$-terminal sequences strikingly diverge between archaeal and eukaryotic species, although still sharing a similar "ribbon" fold, organised like a beta barrel [11]. The model of the human NOP10 3D structure was then superimposed with the frame of the whole H/ACA RNP structure, as observed for archaeal complexes (PDB 2EY4). This superimposition is supported by the fact that the structure of the individual subunits, overall organisation and interfaces are similar between archaeal and yeast $\mathrm{H} /$ ACA RNP complexes [11]. Tyr6 is buried with the small, N-terminal globular, ribbon domain of NOP10 and likely plays a critical for the stability of this domain, in close contact with human Dyskerin DKC1 (Cbf5). In archaeal proteins, the corresponding position is occupied by one of the four cysteine encapsulating a zinc ion within the ribbon domain [11]. The name of the human subunits of the H/ACA RNP complex are given within brackets. Molecular graphics and analyses were performed using the UCSF Chimera package [13]. Models of the three-dimensional structure were built using Modeller 9v15 [14], according to the alignment presented in Lı et al. [11].

In this family, the combination of familial pulmonary fibrosis, the extra-pulmonary manifestations, including haematological and liver disease, the significant telomere shortening, and the genetic anticipation (II.4: 61 years; III.4 onset at 26 years/II.1: 66 years; III.2: 43 years) strongly evokes a diagnosis of telomeropathy [15]. This is supported by the identification of a monoallelic rare variation predicted to be deleterious in the NOP10 telomere-related gene that co-segregates with disease. Collectively, these results provide the first evidence that mono-allelic NOP10 mutation can predispose to short telomere syndrome (familial pulmonary fibrosis, liver and haematological diseases). 
Caroline Kannengiesser $\circledast^{1,2,3,12}$, Effrosyni D. Manali ${ }^{4,12}$, Patrick Revy ${ }^{5}$, Isabelle Callebaut ${ }^{6}$, Ibrahima $\mathrm{Ba}^{1,2,3}$, Adrien Borgel $^{1,2}$, Claire Oudin ${ }^{1,2}$, Aggeliki Haritou ${ }^{4,7}$, Lykourgos Kolilekas ${ }^{8}$, Katerina Malagari ${ }^{\prime}$, Raphael Borie $\oplus^{3,10}$, Elodie Lainey ${ }^{11}$, Catherine Boileau $\oplus^{1,2}$, Bruno Crestani $^{10,3,12}$ and Spyros A. Papiris ${ }^{4,12}$

${ }^{1}$ APHP Service de Génétique, Hôpital Bichat, Paris, France. ${ }^{2}$ Université Paris Diderot, Sorbonne Paris Cité, Paris, France. ${ }^{3}$ INSERM UMR 1152, Université de Paris, Paris, France. ${ }^{4}$ 2nd Pulmonary Medicine Dept, General University Hospital "Attikon", Medical School, National and Kapodistrian University of Athens, Athens, Greece. ${ }^{5}$ INSERM UMR 1163, Laboratory of Genome Dynamics in the Immune System, labéllisé Ligue contre le cancer, Paris DescartesSorbonne Paris Cité University, Imagine Institute, Paris, France. ${ }^{6}$ Sorbonne Université, Muséum National d'Histoire Naturelle, UMR CNRS 7590, Institut de Minéralogie, de Physique des Matériaux et de Cosmochimie, IMPMC, Paris, France. ${ }^{7}$ Ioannina, Greece. ${ }^{8} 7$ th Pulmonary Dept, Athens Chest Hospital "Sotiria", Athens, Greece. ${ }^{9}$ 2nd Dept of Radiology, General University Hospital "Attikon", Medical School, National and Kapodistrian University of Athens, Athens, Greece. ${ }^{10}$ APHP, Hôpital Bichat, Service de Pneumologie A, DHU FIRE, Centre de référence des maladies pulmonaires rares, Paris, France. ${ }^{11}$ Service d'Hématologie Biologique, Hôpital Robert Debré- APHP, UMRS_1131Institut Universitaire d'Hématologie, Paris, France. ${ }^{12}$ These authors contributed equally to this work.

Correspondence: Effrosyni D. Manali, Assistant General University Hospital “Attikon”, Medical School, National and Kapodistrian University of Athens, Greece. E-mail: fmanali@otenet.gr

Received: 21 Dec 2019 | Accepted after revision: 10 Feb 2020

Conflict of interest: C. Kannengiesser has nothing to disclose. E.D. Manali reports personal fees and non-financial support from Boehringer Ingelheim and Roche, during the conduct of the study. P. Revy has nothing to disclose. I. Callebaut has nothing to disclose. I. Ba has nothing to disclose. A. Borgel has nothing to disclose. C. Oudin has nothing to disclose. A. Haritou has nothing to disclose. L. Kolilekas has nothing to disclose. K. Malagari has nothing to disclose. R. Borie reports grants and personal fees from Boehringer Ingelheim and Roche, personal fees from Savapharma, outside the submitted work. E. Lainey has nothing to disclose. C. Boileau has nothing to disclose. B. Crestani reports personal fees from AstraZeneca and Sanofi, grants, personal fees and non-financial support from Boehringer Ingelheim and Roche, personal fees and non-financial support from BMS, outside the submitted work. S.A. Papiris reports personal fees and non-financial support from Boehringer Ingelheim and Roche, during the conduct of the study.

Support statement: This work (exome sequencing) was supported by a grant "FPI-SPC" from Université Sorbonne Paris Cité (B. Crestani) and the Chancellerie des Universités de Paris (legs Poix; C. Kannengiesser). Funding information for this article has been deposited with the Crossref Funder Registry.

\section{References}

1 Kropski JA, Blackwell TS, Loyd JE. The genetic basis of idiopathic pulmonary fibrosis. Eur Respir J 2015; 45: $1717-1727$.

2 Alder JK, Hanumanthu VS, Strong MA, et al. Diagnostic utility of telomere length testing in a hospital-based setting. Proc Natl Acad Sci USA 2018; 115: E2358-E2365.

3 Niewisch MR, Savage SA. An update on the biology and management of dyskeratosis congenita and related telomere biology disorders. Expert Rev Hematol 2019; 12: 1037-1052.

4 Armanios M, Blackburn EH. The telomere syndromes. Nat Rev Genet 2012; 13: 693-704.

5 Walne AJ, Vulliamy T, Marrone A, et al. Genetic heterogeneity in autosomal recessive dyskeratosis congenita with one subtype due to mutations in the telomerase-associated protein NOP10. Hum Mol Genet 2007; 16: 1619-1629.

6 Richards S, Aziz N, Bale S, et al. Standards and guidelines for the interpretation of sequence variants: a joint consensus recommendation of the American College of Medical Genetics and Genomics and the Association for Molecular Pathology. Genet Med 2015; 17: 405-424.

7 Borie R, Tabèze L, Thabut G, et al. Prevalence and characteristics of TERT and TERC mutations in suspected genetic pulmonary fibrosis. Eur Respir J 2016; 48: 1721-1731.

8 Gable DL, Gaysinskaya V, Atik CC, et al. ZCCHC8, the nuclear exosome targeting component, is mutated in familial pulmonary fibrosis and is required for telomerase RNA maturation. Genes Dev 2019; 33: 1381-1396.

9 Borie R, Bouvry D, Cottin V, et al. Regulator of telomere length 1 (RTEL1) mutations are associated with heterogeneous pulmonary and extra-pulmonary phenotypes. Eur Respir J 2019; 53: 1800508.

10 Collopy LC, Walne AJ, Cardoso S, et al. Triallelic and epigenetic-like inheritance in human disorders of telomerase. Blood 2015; 126: 176-184.

11 Li S, Duan J, Li D, et al. Reconstitution and structural analysis of the yeast box H/ACA RNA guided pseudouridine synthase. Genes Dev 2011; 25: 2409-2421.

12 Rashid R, Liang B, Baker DL, et al. Crystal structure of a Cbf5-Nop10-Garl complex and implications in RNA-guided pseudouridylation and diskeratosis congenita. Mol Cell 2006; 21: 249-260.

13 Pettersen EF, Goddard TD, Huang CC, et al. UCSF Chimera-a visualization system for exploratory research and analysis. J Comput Chem 2004; 25: 1605-1612.

14 Marti-Renom MA, Stuart A, Fiser A, et al. Comparative protein structure modeling of genes and genomes. Annu Rev Biophys Biomol Struct 2000; 29: 291-325.

15 Borie R, Le Guen P, Ghanem M, et al. The genetics of interstitial lung diseases. Eur Respir Rev 2019; 28: 190053. 